\title{
Low expression of microRNA-320b correlates with tumorigenesis and unfavorable prognosis in glioma
}

\author{
QIAO-LI LV ${ }^{1}$, HONG DU ${ }^{2}$, YAN-LING LIU ${ }^{3}$, YUANG-TAO HUANG ${ }^{4}$, GUI-HUA WANG ${ }^{5}$, \\ XUE ZHANG ${ }^{3}$, SHU-HUI CHEN ${ }^{6}$ and HONG-HAO ZHOU ${ }^{3}$ \\ ${ }^{1}$ Department of Science and Education, Jiangxi Key Laboratory of Translational Cancer Research, \\ Jiangxi Cancer Hospital, Nanchang, Jiangxi 330029; ${ }^{2}$ Department of Pharmacy, Qingdao Mental Health Center, \\ Qingdao, Shandong 266034; ${ }^{3}$ Department of Clinical Pharmacology, Xiangya Hospital, Central South University; \\ ${ }^{4}$ Department of Neurology, The Brain Hospital of Hunan Province; ${ }^{5}$ Department of Oncology, \\ Changsha Central Hospital, Changsha, Hunan 410008; ${ }^{6}$ Department of Radiation Oncology, \\ Jiangxi Cancer Hospital, Nanchang, Jiangxi 330029, P.R. China
}

Received October 2, 2016; Accepted March 13, 2017

DOI: $10.3892 /$ or.2017.5762

\begin{abstract}
Accumulating evidence demonstrates that dysregulated microRNAs (miRNAs) play a critical role in tumorigenesis and progression of various cancers. miR-320b, a member of miR-320 family, was revealed downregulated in numerous human cancers, including nasopharyngeal carcinoma and colorectal cancer. However, the function of miR-320b in human glioma remained poorly defined. In this study, we report that miR-320b was lowly expressed in glioma tissues and cell lines in contrast with controls, being closely correlated with histological malignancy of glioma. Furthermore, patients with low expression of miR-320b were associated with poor prognostic outcomes. In vitro functional assays indicated that overexpression of miR-320b could markedly enhance cell apoptosis rate and suppress cell proliferation, migration and invasion. miR-320b mimic impaired cell cycle and metastasis through inhibiting the expression of G1/S transition key regulator Cyclin D1 as well as decreasing the expression level of MMP2 and MMP9. Additionally, upregulation of miR-320b could markedly promote apoptosis by increasing the level of Bax and reducing Bcl-2 expression in glioma. Taken together, our data suggested that miR-320b
\end{abstract}

Correspondence to: Professor Hong-Hao Zhou, Department of Clinical Pharmacology, Xiangya Hospital, Central South University, 110 Xiang Ya Road, Changsha, Hunan 410008, P.R. China E-mail: hhzhou2003@126.com

Professor Shu-Hui Chen, Department of Radiation Oncology, Jiangxi Cancer Hospital, 519 Beijing East Road, Nanchang, Jiangxi 330029, P.R. China

E-mail: chenshuhui2008@126.com

Key words: miR-320b, cell cycle, proliferation, metastasis, apoptosis, glioma might serve as a novel prognostic marker and potential therapeutic target for glioma.

\section{Introduction}

Glioma is one of the most common malignant tumor in adult central nervous system (CNS) (1). Despite recent advances in combination therapy, which consists mainly of surgical resection, chemotherapy and radiotherapy, the median survival of glioblastoma remains at merely 15-17 months $(2,3)$. Therefore, it is urgent to find novel biomarkers that can identify the biological characteristics of tumors and predict the prognosis of patients with glioma (4). miRNAs are single-stranded non-coding RNAs and usually 18-25 nucleotides in length. Mostly miRNAs are combined with the $3^{\prime}$ end of the untranslated regions (3'UTR) of target mRNAs, resulting in their degradation or inhibition of translation (5). Importantly, miRNAs have acquired extensive attention recently as important regulators in a wide range of biological processes, such as immune response (6), angiogenesis (7), differentiation (8), cell proliferation and metastasis $(9,10)$. Emerging studies have shown that dysreglated miRNAs function as either carcinogenic factors or tumor suppressors in a variety of cancers, such as miR-575 promoting growth and invasion of non-small cell lung cancer (11), miR-582-5p inhibiting proliferation of hepatocellular carcinoma by targeting CDK1 and AKT3 (12), miR-22 acting as an antitumor gatekeeper in de novo acute myeloid leukemia (AML) (13).

miRNA-320b has been identified to play a suppressive role in various tumors, including nasopharyngeal carcinoma (14) and colorectal cancer (15). However, the implication of miR-320b in glioma is still unclear. In our study, we evaluated the level of miR-320b in glioma tissues as well as in normal brain tissues, and explored the correlation between miR-320b with clinical characteristics and prognosis of glioma patients. Furthermore, using miRNA-320b mimics, we investigated the potential molecular mechanisms of miR-320b involved in 
proliferation, apoptosis, migration and invasion in glioma U87 and U251 cells.

\section{Materials and methods}

Tissue samples. Our study was approved by the Ethics Committee of The First Affiliated Hospital of Nanchang University (ethics approval no. 2010-015; date, 12 March 2010), with written informed consent obtained from all the patients. The glioma tissues in this study were obtained from 65 patients who had undergone tumor resection without radiotherapy or chemotherapy from 2010 to 2013 at The First Affiliated Hospital of Nanchang University (Nanchang, Jiangxi, China). Sixteen normal brain tissues from cerebral trauma patients or epilepsy patients were used as controls. All samples were immediately frozen in liquid nitrogen and stored at $-80^{\circ} \mathrm{C}$ until RNA extraction. Each patient has experienced a follow-up period of up to 48 months since surgical resection.

Cell lines and culture conditions. The human glioma cells (U87, U251 and T98G) were purchased from the Cell Bank of the Shanghai Branch of Chinese Academy of Sciences and cultured in Dulbecco's modified Eagle's medium (DMEM) with $10 \%$ fetal bovine serum (FBS, Gibco, Carlsbad, CA, USA). All the cells were incubated at $37^{\circ} \mathrm{C}$ in a humidified atmosphere of $5.0 \% \mathrm{CO}_{2}$.

$R N A$ extraction and $q R T-P C R$. Total RNA was isolated from tissue samples and cell lines with TRIzol reagent (Invitrogen, Carlsbad, CA, USA) following the manufacturer's instructions. The isolated RNA was reverse transcribed into cDNA using Thermo Scientife RevertAid First Strand cDNA Sythesis kit (Thermo Fisher Scientific). The expression was qualified by qRT-PCR using SYBR Green real-time PCR kit (Takara, Dalian, China) on a LightCycler 480 (Roche, USA), with U6 as a normalizing control and $2^{-\Delta \mathrm{Ct}}$ as the calculation method. The forward primers and reverse primers for miR-320b and U6 were purchased from Ribobio (Guangzhou, China).

Western blot analysis. Glioma tissues and cell lines were lysed using RIPA buffer (Beyotime Institute of Biotechnology, Shanghai, China) and protein concentration was quantified with BCA Protein assay kit (Thermo, USA). The proteins were separated by $10 \%$ SDS denaturing polyacrylamide gel (SDS-PAGE) and then transferred onto a polyvinylidene fluoride (PVDF) membrane. Then, the primary antibody to MMP2, MMP9, Cyclin D1, Bax, Bcl-2 or $\beta$-actin was incubated with the membranes overnight at $4^{\circ} \mathrm{C}$. After being washed three times with TBST, the membranes were incubated with horseradish peroxidase-conjugated secondary antibody for $2 \mathrm{~h}$. The protein bands were visualized with enhanced chemiluminescence (ECL, GE Healthcare, USA), with their images captured by Quantity One software (BioRad, USA). The levels of proteins were represented by the relative densities of their bands normalized with those of $\beta$-actin. Antibodies against MMP2, MMP9, Cyclin D1, Bax and Bcl-2 were obtained from CST Biotech (USA). Antibody to $\beta$-actin from Sigma (USA) was adopted as a loading control.
Cell proliferation assay. The effect of miR-320b on the proliferation ability of U87 and U251 cells was determined by MTS (CellTiter 96 aqueous one solution reagent; Promega, Madison, WI, USA) according to the manufacturer's instructions. U87 and U251 cells lines were cultured in 96-well plates ( $3 \times 10^{3} /$ well) and their proliferation was assessed at $490 \mathrm{~nm}$ on a microplate reader (Bio-Rad Laboratories, Inc.) every $24 \mathrm{~h}$ to $72 \mathrm{~h}$ after transfection. Each experiment was repeated at least three times independently.

Colony formation assay. Briefly, cells (1,000/well) were seeded into 6-well plates and cultured for 15 days. Then colonies were fixed for 10 min with $4 \%$ paraformaldehyde and stained with $0.1 \%$ crystal violet (Beyotime Institute of Biotechnology) for $30 \mathrm{~min}$. The number of colonies was counted under a microscope and stained colonies were photographed using a high-resolution camera. The experiment was performed at least in triplicate.

Flow cytometry analysis of cell cycle and apoptosis. For cell cycle analysis, all cells were harvested by trypsinization, washed with PBS and then fixed with ice-cold $70 \%$ ethanol at $-20^{\circ} \mathrm{C}$ overnight. Fixed cells were then suspended in $300 \mu \mathrm{l}$ staining buffer, $10 \mu 1$ propidium iodide (PI) and $5 \mu 1$ RNase (Beyotime Institute of Biotechnology) for $20 \mathrm{~min}$ at room temperature in the dark, followed by the examination on a FC500 flow cytometer (Beckman Coulter).

Cell apoptosis was detected using Annexin V-FITC/propidium iodide (PI) Apoptosis Detection kit (BD Biosciences, San Jose, CA, USA) according to the manufacturer's instructions. Cells were transfected with miR-320b mimics for $48 \mathrm{~h}$ and harvested by trypsinization before being washed with ice-cold PBS and suspended with $195 \mu 1$ binding buffer. Annexin V-FITC $(5 \mu \mathrm{l})$ and $5 \mu \mathrm{l}$ of PI were subsequently added to the suspension and the cells were mixed for additional $15 \mathrm{~min}$ at room temperature in the dark. The percentage of apoptotic cells was measured by FC500 flow cytometer, and data analysis was performed with FlowJo software. The experiments were performed independently three times for each cell line.

Wound healing assay. Wound healing assay was used to detect cell migration. In brief, U87 and U251 cells were cultured in 6 -well plates until they reached $90 \%$ confluence with cell layers scratched using a $200 \mu 1$ plastic pipette tip to form wounded gaps. Then the detached cells were washed with PBS for three times and cultured in a medium containing $2 \%$ FBS at $37^{\circ} \mathrm{C}$. Representative scrape lines were imaged, along which different stages of wound healing were observed. At least three separate experiments were carried out.

Transwell invasion assay. Transwell invasion assay was used to determine cell invasion. U87 and U251 cells transfected with mimic of miR-320b or non-specific mimic (NC) were resuspended $\left(1 \times 10^{5}\right.$ cells $\left./ \mathrm{ml}\right)$ in $200 \mu \mathrm{l}$ serum of $1 \%$ FBS medium and were added on a filter coated with basement membrane Matrigel (BD Bioscience, Bedford, MA, USA) according to the manufacturer's protocol. After $24 \mathrm{~h}$ of incubation, the cells and Matrigel on the upper membrane surface were removed gently with cotton swabs, and invading cells on the lower 

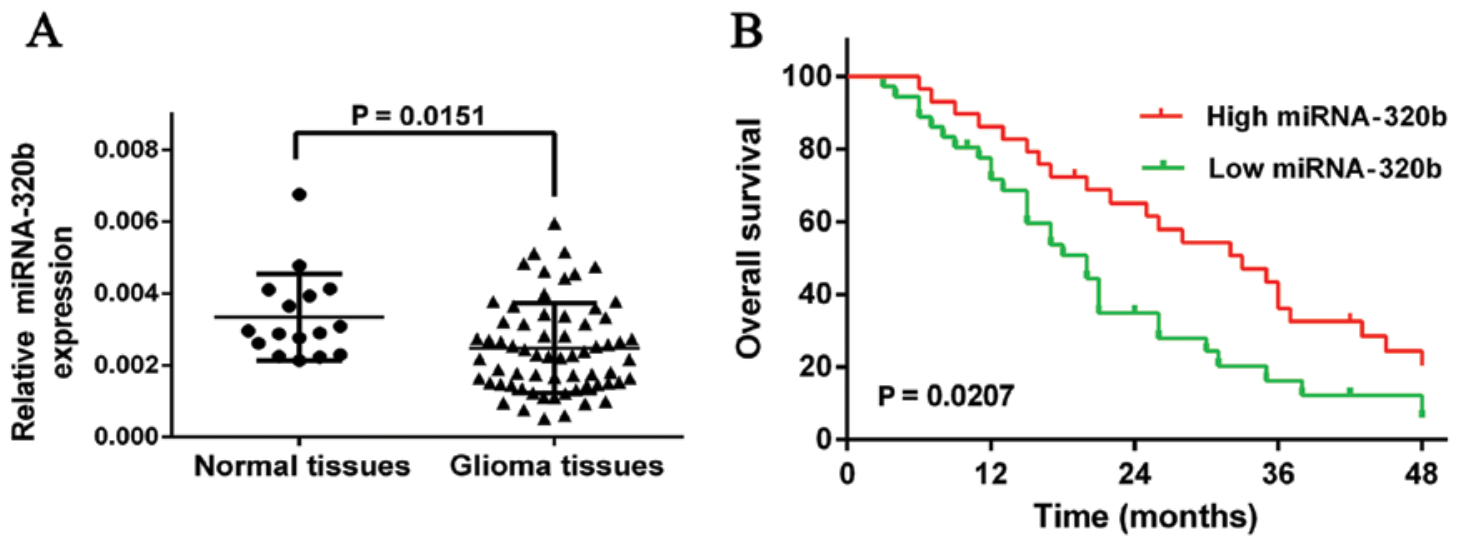

Figure 1. Relative expression of miR-320b in glioma tissues and its clinical significance. (A) The expression level of miR-320b was calculated in 16 normal brain tissues and 65 glioma tissues. (B) With the median value as a reference, patients with glioma were divided into miR-320b high and low expression group, patients with low expression of miR-320b were associated with a poor overall survival in glioma ( $\mathrm{P}=0.0207$, Kaplan-Meier method).
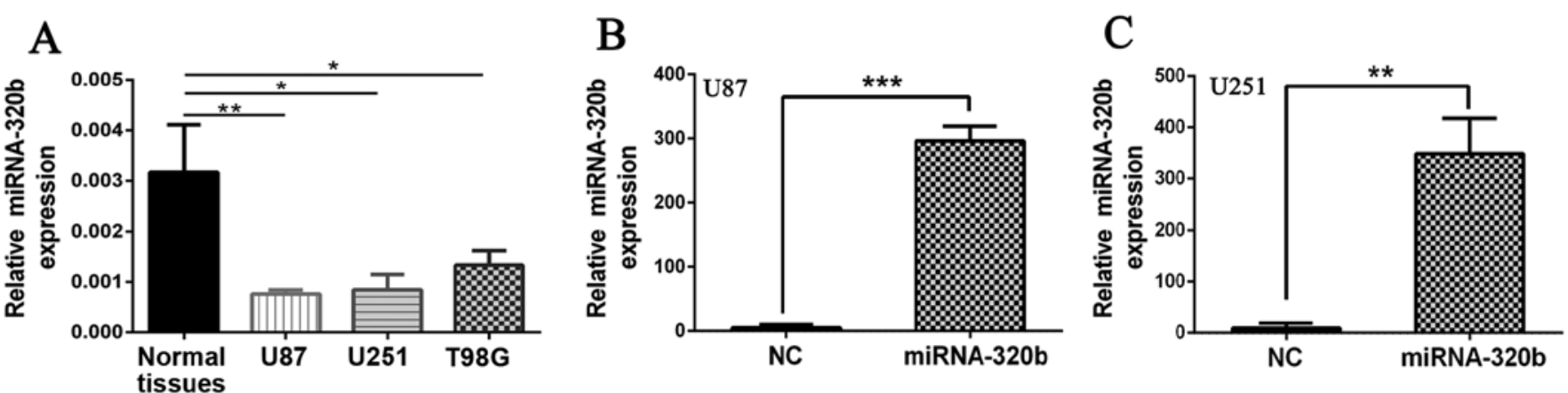

Figure 2. Expression levels of miR-320b in glioma cell lines and mimic efficiency. (A) The expression of miR-320b in glioma cell lines was significantly lower compared with normal brain tissues. (B and C) miR-320b mimic efficiency was determined by qRT-PCR in U87 and U251 cells $\left({ }^{*} \mathrm{P}<0.05,{ }^{* *} \mathrm{P}<0.01,{ }^{* * *} \mathrm{P}<0.001\right)$.

surface were fixed with $4 \%$ paraformaldehyde for $10 \mathrm{~min}$ and stained with crystal violet for $30 \mathrm{~min}$. The invading cells were photographed in randomly selected fields and counted using a light microscope. The experiments for each group were performed in triplicate and the results were averaged.

Statistical analysis. Statistical analysis was performed with SPSS 20.0 software system (IBM, SPSS, Chicago, IL, USA). Data on the relative expression of miRNA were reported as means $\pm \mathrm{SD}$ and Student's t-test was used to analyze differences between groups. A Chi-square test was applied to determine the association of miR-320b levels with clinicopathologic features. Survival analysis was carried out with the Graphpad 6. The hazard ratio (HR) was estimated via a Cox regression model and overall survival curves were plotted from Kaplan-Meier estimates, while log-rank test was conducted to compare the survival distributions between two groups. Differences were considered statistically significant at $\mathrm{P}<0.05$.

\section{Results}

miR-320b is significantly downregulated in glioma tissues. To explore the implication of miRNA-320b in the development and progression of glioma, the expression levels of miR-320b in 65 glioma patients without radiotherapy or chemotherapy were compared against those of 16 normal brain tissues by qRT-PCR. The results demonstrated that miR-320b was significantly downregulated in glioma tissues compared with normal tissues (Fig. 1A, $\mathrm{P}=0.0151$ ).

Low expression of miR-320b was associated with poor prognosis of glioma patients. In order to further investigate the association of miRNA-320b expression with prognosis of glioma patients, Kaplan-Meier analysis and log-rank test were used to evaluate the effects of miR-320b expression on overall survival, discovering that patients with low expression of miR-320b might have a worse overall survival compared with those with highly expressed miR-320b (Fig. 1B, HR, 1.853; 95\% CI, 1.137-3.464; $\mathrm{P}=0.0207$ ).

Correlations of miR-320b expression with clinicopathological features of glioma patients. To further elucidate the relationship between miR-320b with clinical characteristics of glioma patients, 65 glioma patients were divided into two groups, low miR-320b expression $(\mathrm{n}=36)$ and high miR-320b expression $(n=29)$. Our data revealed that the expression levels of miR-320b was greatly related to tumor grade of glioma $(\mathrm{P}=0.0070)$, while not in association with age, gender and tumor location (Table I, $\mathrm{P}=0.6781,0.3437$ and 0.7849 , respectively). Thus, we speculated that miR-320b might play important roles in the progression of glioma. 
Table I. Correlation between miR-320b expression and clinicopathological features of glioma patients.

\begin{tabular}{|c|c|c|c|c|}
\hline \multirow[b]{2}{*}{ Clinical characteristic } & \multirow[b]{2}{*}{ No. of patients } & \multicolumn{2}{|c|}{ No. of patients } & \multirow[b]{2}{*}{ P-value } \\
\hline & & High expression (29) & Low expression (36) & \\
\hline \multicolumn{5}{|l|}{ Age (years) } \\
\hline$<45$ & 31 & 13 & 18 & 0.6781 \\
\hline$\geq 45$ & 34 & 16 & 18 & \\
\hline \multicolumn{5}{|l|}{ Gender } \\
\hline Male & 40 & 16 & 24 & 0.3437 \\
\hline Female & 25 & 13 & 12 & \\
\hline \multicolumn{5}{|l|}{ Clinical stage } \\
\hline Low grades I-II & 35 & 21 & 14 & 0.0070 \\
\hline High grades III - IV & 30 & 8 & 22 & \\
\hline \multicolumn{5}{|l|}{ Tumor location } \\
\hline Frontal & 19 & 7 & 12 & 0.7849 \\
\hline Parietal & 6 & 2 & 4 & \\
\hline Occipital & 11 & 6 & 5 & \\
\hline Temporal & 12 & 5 & 7 & \\
\hline Others & 17 & 9 & 8 & \\
\hline
\end{tabular}

Significant overexpression of miR-320b with mimics in U87 and U251. To identify whether miR-320b was associated with the progression of glioma, function analyses were conducted in vitro. First, we performed qRT-PCR to calculated the expression of miR-320b in three glioma cell lines (U87, U251, T98G), finding that similar to the situation for the glioma tissues, miR-320b were markedly lower in glioma cell lines (U87, U251 and T98G) compared with normal brain tissues (Fig. 2A). Then, mimic of miR-320b and miR-NC were transfected into U87 and U251 cells which had the lowest levels of miRNA-320b. As shown in Fig. 2B and C, miR-320b was effectively overexpressed in U87 and U251 cell lines compared with $\mathrm{NC}$ groups (Fig. 2B, $\mathrm{P}<0.001$ for $\mathrm{U} 87$; $2 \mathrm{C}, \mathrm{P}<0.001$ for U251).

miR-320b mimic inhibits U87 and U251 cell proliferation. In order to further determine the role of miR-320b in glioma cells, MTS and colony formation assays were carried out in cultured U87 and U251 cells. MTS results suggested that overexpression of miR-320b could significantly inhibit cell viability in contrast with $\mathrm{NC}$ groups at $72 \mathrm{~h}$ both in U87 and U251 cell lines (Fig. 3A). Moreover, colony formation assay results demonstrated that the number of U87 and U251 cell colonies was strikingly decreased by the miR-320b mimic compared with the NC groups (Fig. 3B).

Subsequently, to explore the mechanisms underlying proliferation suppression after miR-320b mimic transfection, we examined the effects of miR-320b on cell cycles of U87 and U251 cells by PI staining and flow cytometry. Flow cytometric analysis indicated that miR-320b mimic could significantly induce accumulation of cells in G0/G1 phase and correspondingly decrease the percentage of S phase cells (Fig. 3C). These data suggested that overexpression of miR-320b could inhibit glioma growth and development by suppressing cell cycle progression.
miR-320b mimics inhibit migration and invasion of U87 and U251 cell lines. Furthermore, to investigate the correlation of miR-320b expression with the migration and invasion capabilities of glioma cells, wound healing assay and Transwell invasion assay were conducted. In the wound healing assay, cell migration was inhibited after the transfection with miR-320b mimics and incubation for $24 \mathrm{~h}$ (Fig. 4A). Besides, Transwell assay demonstrated that miR-320b mimics could inhibit invasion abilities of U87 and U251 cells in comparison with NC groups (Fig. 4B).

miR-320b mimic promotes U87 and U251 apoptosis. As defects in apoptosis could induce carcinogenesis and cancer progression, we subsequently investigated whether overexpression of miR-320b could affect apoptosis of glioma cell lines. Flow cytometry analysis was performed and results suggested the significant increase of apoptotic rates of U87 and U251 cells transfected with miR-320b mimics compared with NC groups (Fig. 4C), indicating that miR-320b might play a crucial role in the apoptosis regulation of human glioma cells.

Signaling pathways relative to proliferation, apoptosis, migration and invasion of U87 and U251 cells. To further explore the molecular mechanisms by which miRNA-320b promoted glioma progression, western blot analysis was conducted to detect the target proteins involved in cell cycle, apoptosis, invasion and metastasis of tumor. As shown in Fig. 5, compared with NC groups, the expression of MMP2, MMP9, Cyclin D1 and $\mathrm{Bcl}-2$ was significantly repressed, while the protein level of Bax was elevated significantly in both U87 and U251 cell lines after transfection with miRNA-320b, demonstrating that miRNA-320b probably played a crucial role in the regulation of proliferation, apoptosis, migration and invasion of human glioma cells. 
A

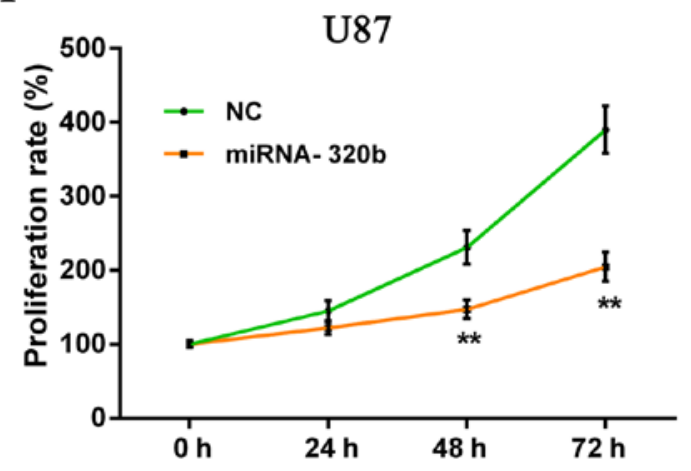

B

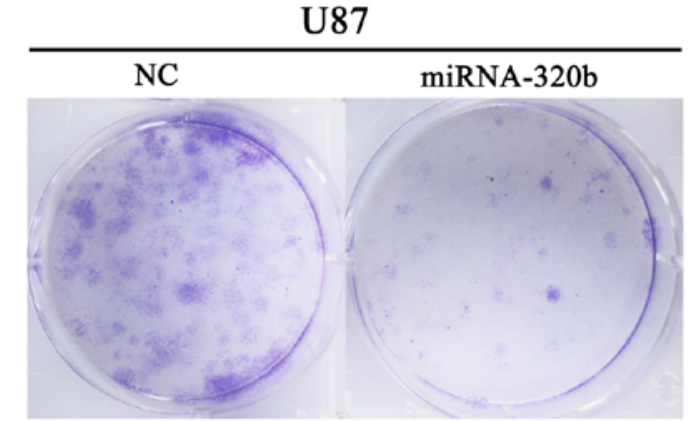

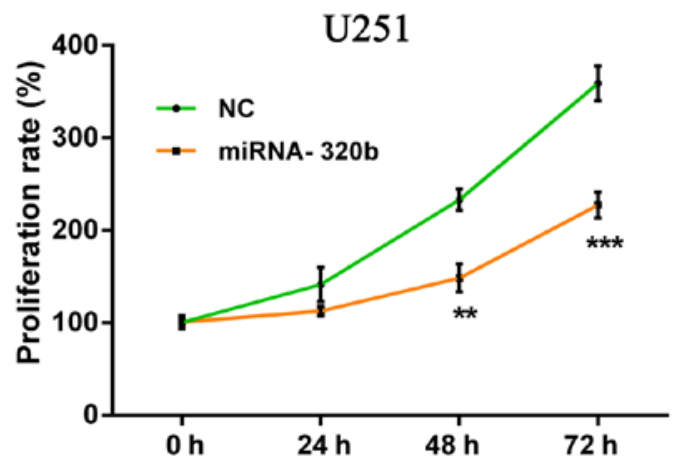

$\mathrm{U} 251$

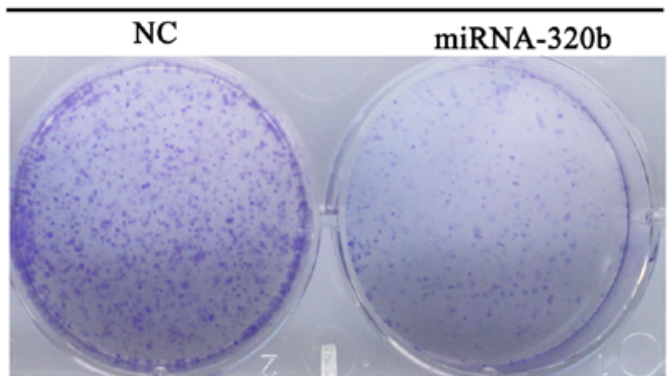

C

U87 NC
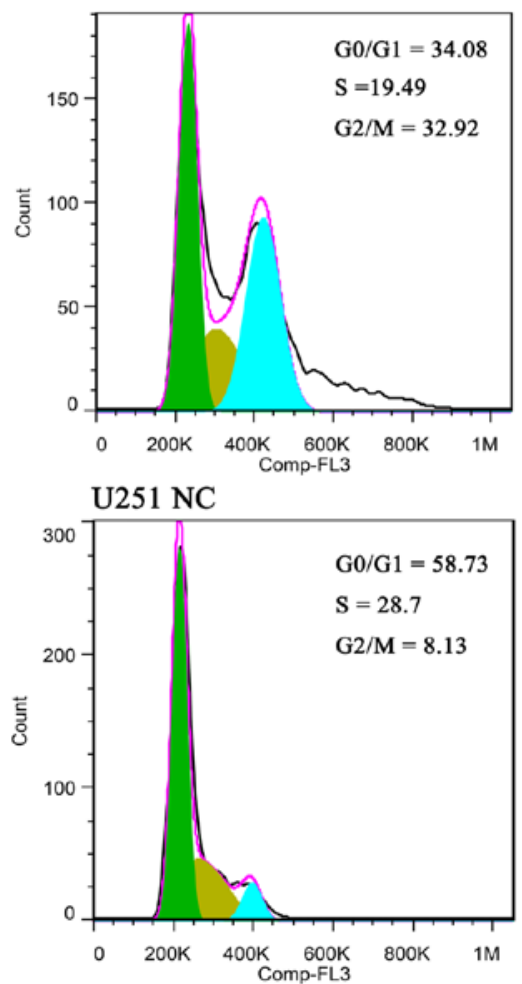

U87 miRNA-320b
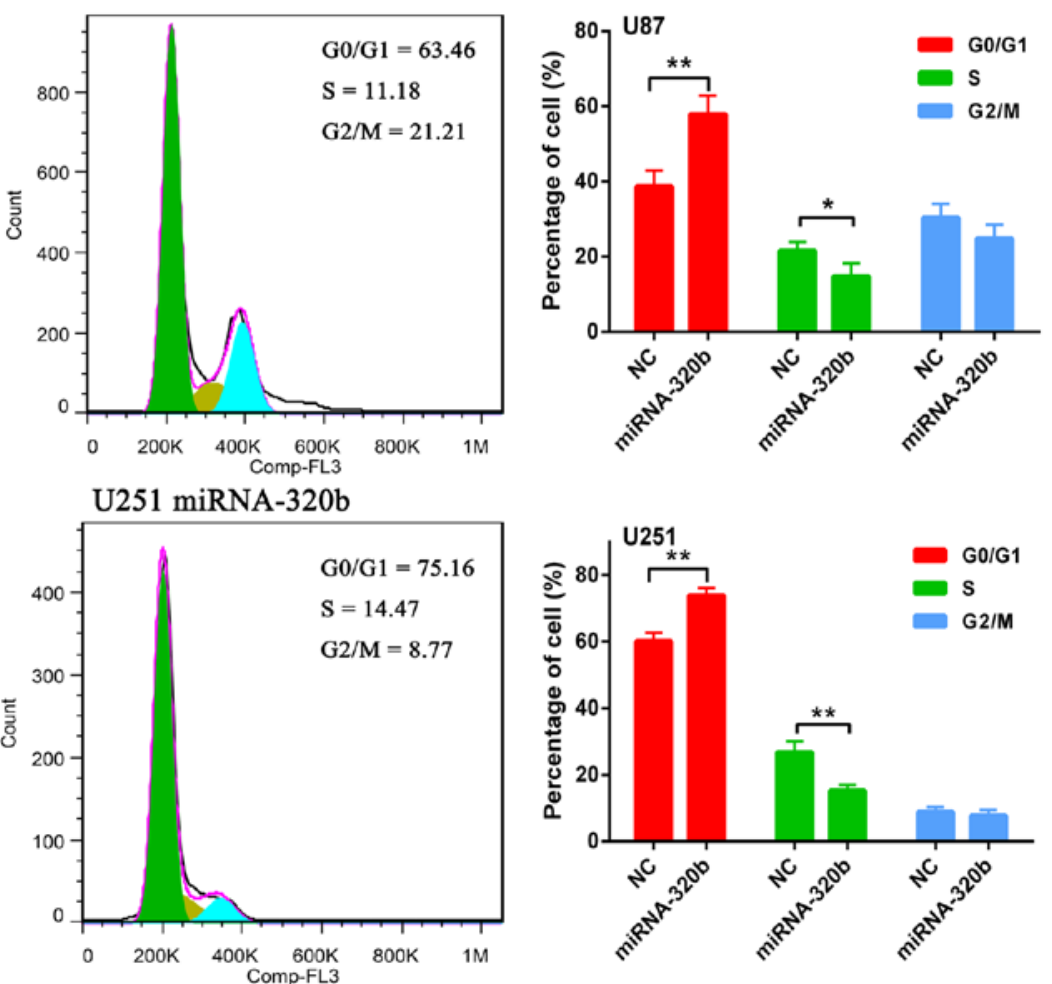

Figure 3. miR-320b mimic inhibits proliferation of glioma cells. (A) MTS assay indicated that overexpression of miR-320b could significantly suppress the proliferation abilities of U87 and U251 cells compared with NC groups at $72 \mathrm{~h}$. (B) Colony formation assay revealed that overexpression of miR-320b markedly reduced the number of colonies of the U87 and U251 cells in comparison with NC groups. (C) Cell cycle analysis showed that overexpression of miR-320b greatly induced the $\mathrm{G} 0 / \mathrm{G} 1$ phase arrest $\left({ }^{*} \mathrm{P}<0.05,{ }^{* *} \mathrm{P}<0.01,{ }^{* * *} \mathrm{P}<0.001\right)$.

\section{Discussion}

A growing number of miRNAs have been demonstrated to be aberrantly expressed in wide spectrum of human diseases, such as Parkinson (16), diabetes (17), atherosclerosis (18), immune diseases (19) and especially malignant tumors (20-24). Recently, dysregulated miRNAs were identified to be crucial in the regulation of carcinogenesis and cancer 
A

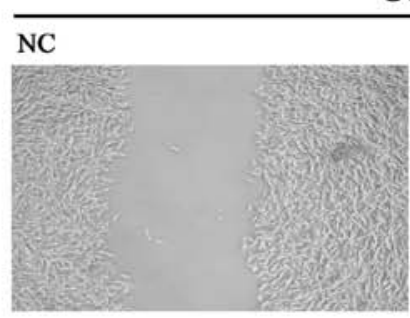

U87

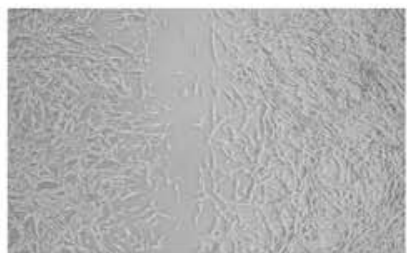

B

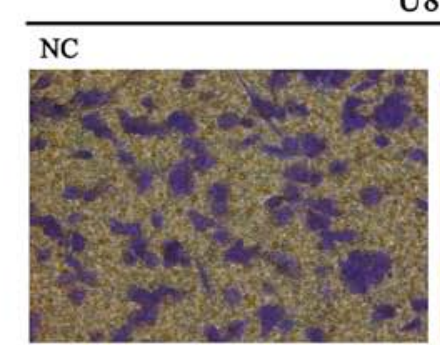

U87

miRNA-320b
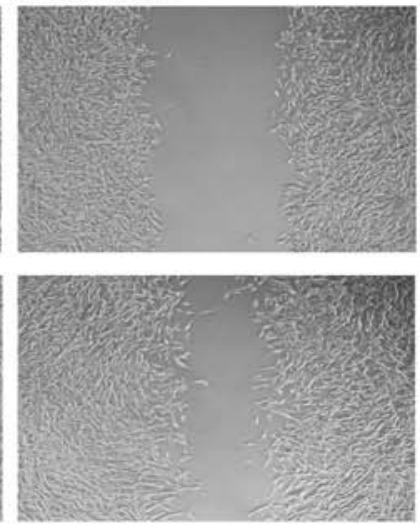

\section{miRNA-320b}

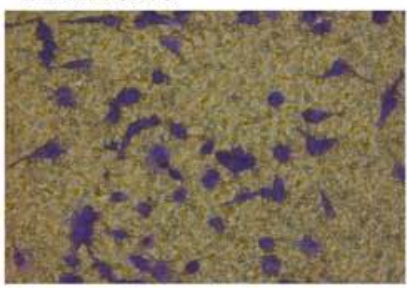

U251
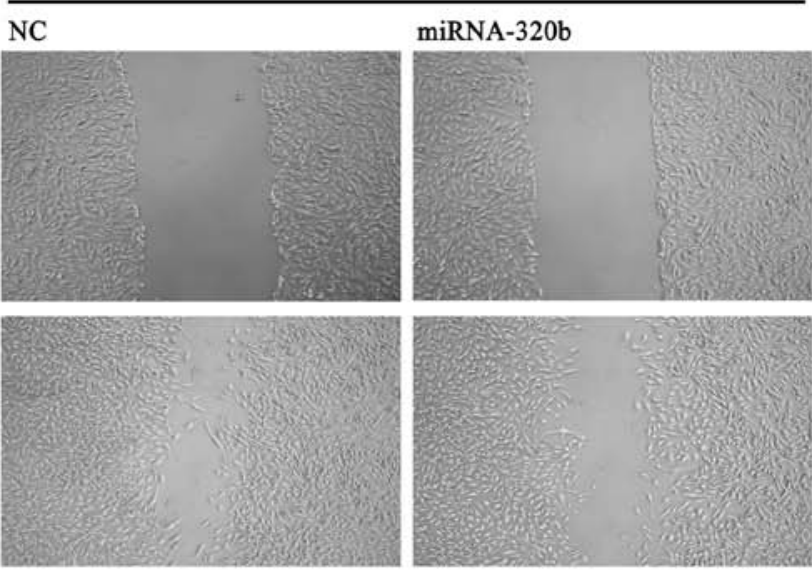

U251

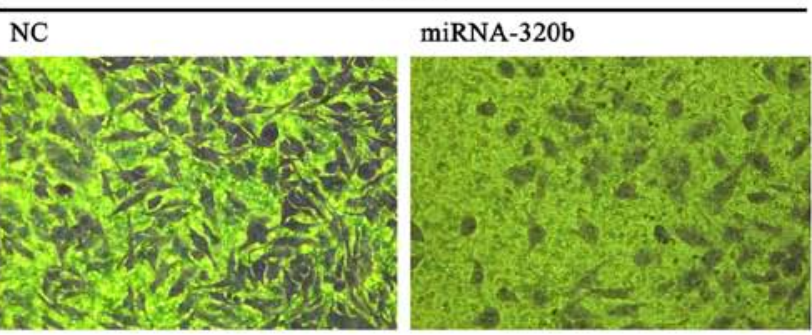

C
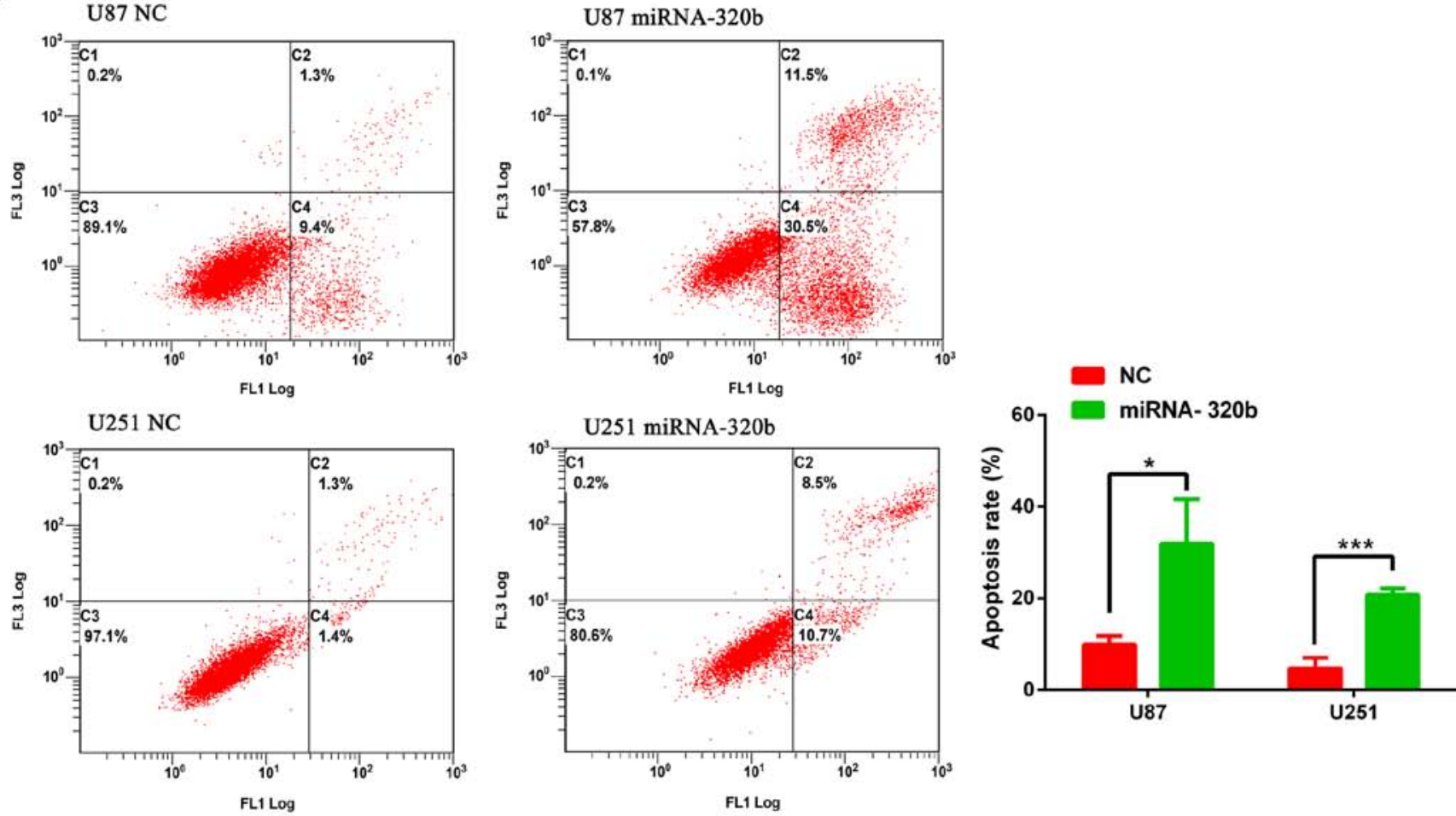

Figure 4. Effects of miR-320b on glioma cell apoptosis, migration and invasion. (A and B) Wound healing assay and Transwell assay indicated that overexpression of miR-320b could significantly inhibit cell migration and invasion in contrast to NC groups in U87 and U251 cells. (C) Annexin V/PI staining and flow cytometry analysis showed that miR-320b markedly enhanced the cell apoptosis rate compared with NC groups in U87 and U251 cells $\left({ }^{*} \mathrm{P}<0.05,{ }^{* * * *} \mathrm{P}<0.001\right)$.

progression (25-27). miR-320b, an important member of miR-320 family, has been reported to be involved in a variety of biological processes. Yan et al found that miR-320b level was significantly lower in prediabetes compared with newly diagnosed type 2 diabetes (T2D) and normal glucose toler- ance (NGT) subjects (28). Zhang et al reported that low serum miR-320b expression could act as a novel indicator of carotid atherosclerosis (29). Moreover, Qin et al provided evidence that elevating the expression of circulating miRNA-320b might serve as a biomarker for unexplained recurrent spontaneous 
A
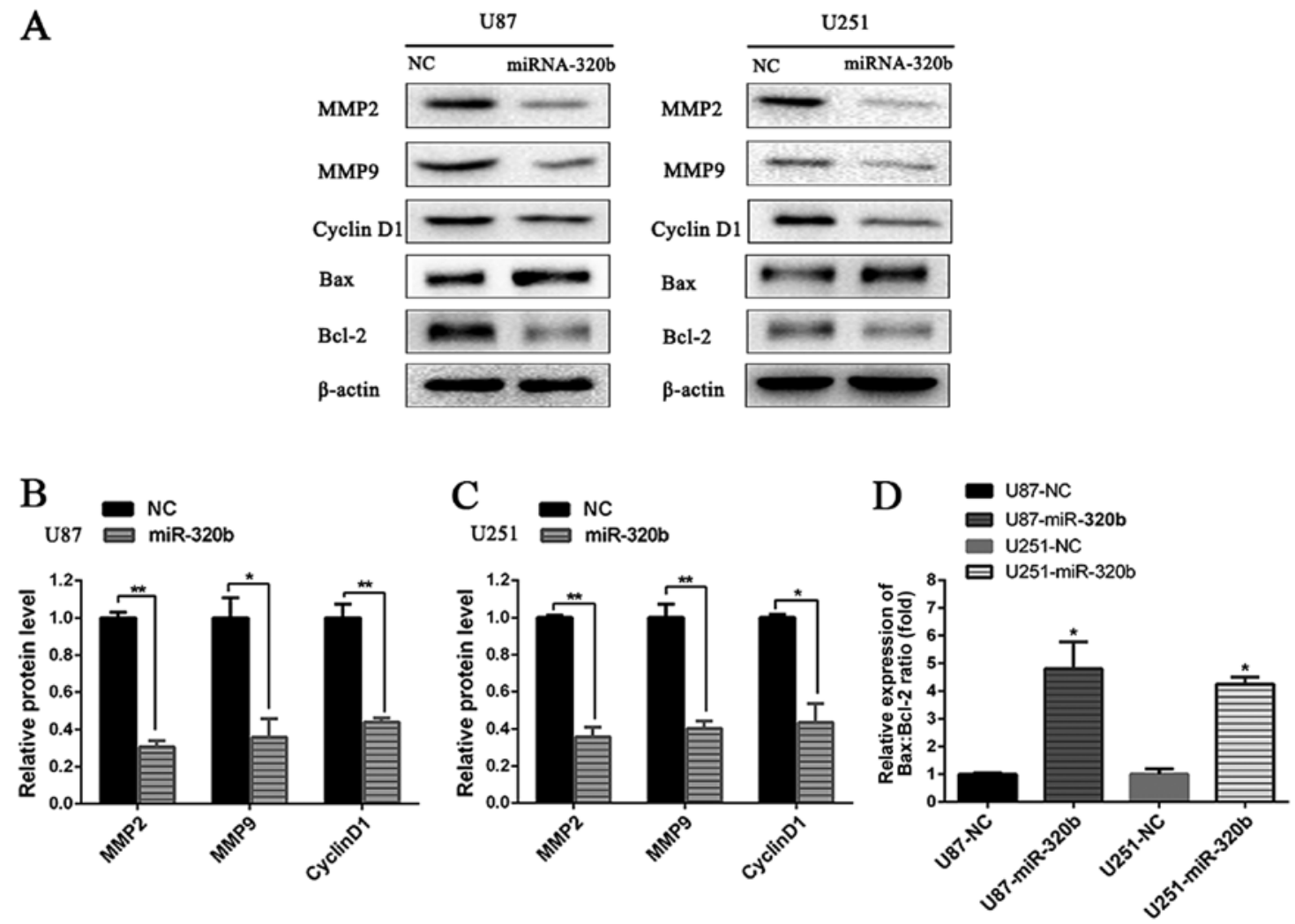

Figure 5. Expression levels of the proteins in cell proliferation, apoptosis, migration and invasion after transfection with miRNA-320b. (A) The expression of MMP2, MMP9, Cyclin D1 and Bcl-2 were significantly decreased, while the expression of Bax was greatly elevated. (B-D) Bar graphs represent the relative expression of the MMP2, MMP9, Cyclin D1 and Bax/Bcl-2 ratio calculated from each group $\left({ }^{*} \mathrm{P}<0.05,{ }^{* *} \mathrm{P}<0.01\right)$.

abortion (URSA) (30). However, limited studies were focused on the role of miRNA-320b as an important regulator in tumor with its pathological role in glioma still poorly understood.

In this study, we investigated the function of miR-320b in glioma, detecting a greatly decreased expression pattern of miR-320b in glioma tissues compared with normal brain tissues as well as in both U87 and U251 glioma cell lines in contrast with NC, which was similar to the results by Tadano et al and Li et al in colorectal adenoma (31) and nasopharyngeal carcinoma patients (14). To further identify the clinical significance of miR-320b, we explored the association between miR-320b and clinicopathological features of glioma patients, finding that its expression was tightly related to tumor clinical stage, with low expression of miR-320b more frequently exhibited in high degree gliomas. Moreover, we detected the correlation of miR-320b expression with overall survival, discovering that glioma patients with decreased expression of miRNA-320b had a worse prognosis of overall survival. These data suggested the involvement of miR-320b in the occurrence and development of glioma. Therefore, functional and pathogenetic studies were conducted in vitro to elucidate its precise role in glioma.

Li et al reported that decrease of miRNA-320b enhanced NPC cell proliferation by targeting TRIAP1 (14). In addition, Wang et al and Tadano et al discovered that miR-320b could markedly promote cell proliferation ability of human colorectal cancer cells by targeting c-Myc and CDK6 both in vitro and in vivo $(15,31)$. Similar results were presented in our study, since MTS and colony formation assays revealed that miRNA320 b mimics significantly suppressed cell proliferation both in U87 and U251 cell lines compared with NC group.
Dysregulation of apoptosis and cell cycle are principal element in the transformation and progression of glioma. Bcl-2 family members, mainly including Bax and Bcl-2, play crucial roles in the process of cancer cell apoptosis. Cyclin D1, usually involved in promotion of tumor growth and regulation of the development of gliomas, is a key regulator of G1/S cell cycle transition. Furthermore, flow cytometric analysis showed that overexpression of $320 \mathrm{~b}$ statistically induced G0/G1 phase arrest and cell apoptosis, and correspondingly reduced the percentage of $S$ phase cells by significantly repressing the expression of Cyclin D1 and BCL-2 and upregulating Bax level. Concordant with our results, Wang et al discovered that miR-320b suppressed cell proliferation by accumulating of cells in G1 phase both in HCT-116 and SW-480 cells (15). Actually, the function of miR-320b in the cell cycle and apoptosis was not clarified in our study, which needs to be elucidated in the future.

Previous studies have shown that all kinds of secreted factors are also very important in the promotion of tumor metastasis. As important secreted proteins MMPs, MMP2 and MMP9 play prominent roles in invasion and migration in the context of malignancy. In this study, we observed that miR-320b mimic could suppress glioma cell migration and invasion through significantly decreasing the expression of MMP2 and MMP9 in contrast with NC groups in both U87 and U251 cells. In colorectal cancer, similar inhibitory effects of miR-320b were observed in SW620 cells and RKO cells by targeting $\beta$-catenin, Neuropilin-1 and Rac-1, resulting in inhibition of proliferation and invasion by competing with its homologous microRNA-320a (32). 
In conclusion, for the first time, our study demonstrated that miR-320b was greatly downregulated in glioma tissues and correlated with poor prognostic outcome of glioma patients. Furthermore, overexpression of miR-320b by mimics markedly inhibited proliferation, invasion and migration, as well as promoted apoptosis of glioma cells. All these findings suggested that miR-320b played a pivotal role in the regulation of glioma progression and could probably be used as an underlying prognostic marker and a novel therapeutic target for gliomas in the future.

\section{Acknowledgements}

This study was supported by The National Natural Science Foundation of China (no. 81503166).

\section{References}

1. Sathornsumetee S and Rich JN: New treatment strategies for malignant gliomas. Expert Rev Anticancer Ther 6: 1087-1104, 2006.

2. Preusser M, Lim M, Hafler DA, Reardon DA and Sampson JH: Prospects of immune checkpoint modulators in the treatment of glioblastoma. Nat Rev Neurol 11: 504-514, 2015.

3. Floyd JA, Galperin A and Ratner BD: Drug encapsulated polymeric microspheres for intracranial tumor therapy: A review of the literature. Adv Drug Deliv Rev 91: 23-37, 2015.

4. Westphal M and Lamszus K: Circulating biomarkers for gliomas. Nat Rev Neurol 11: 556-566, 2015.

5. Jonas $\mathrm{S}$ and Izaurralde E: Towards a molecular understanding of microRNA-mediated gene silencing. Nat Rev Genet 16: 421-433, 2015.

6. Lu LF, Gasteiger G, Yu IS, Chaudhry A, Hsin JP, Lu Y, Bos PD, Lin LL, Zawislak CL, Cho S, et al: A Single miRNA-mRNA interaction affects the immune response in a context- and cell-type-specific manner. Immunity 43: 52-64, 2015.

7. Zheng L, Pu J, Qi T, Qi M, Li D, Xiang X, Huang K and Tong Q: miRNA-145 targets v-ets erythroblastosis virus E26 oncogene homolog 1 to suppress the invasion, metastasis, and angiogenesis of gastric cancer cells. Mol Cancer Res 11: 182-193, 2013.

8. Yuan Z, Ding S, Yan M, Zhu X, Liu L, Tan S, Jin Y, Sun Y, Li Y and Huang T: Variability of miRNA expression during the differentiation of human embryonic stem cells into retinal pigment epithelial cells. Gene 569: 239-249, 2015.

9. Yu G, Li H, Wang J, Gumireddy K, Li A, Yao W, Tang K, Xiao W, $\mathrm{Hu}$ J, Xiao H, et al: miRNA-34a suppresses cell proliferation and metastasis by targeting CD44 in human renal carcinoma cells. J Urol 192: 1229-1237, 2014.

10. Hur K, Toiyama Y, Takahashi M, Balaguer F, Nagasaka T, Koike J, Hemmi H, Koi M, Boland CR and Goel A: MicroRNA$200 \mathrm{c}$ modulates epithelial-to-mesenchymal transition (EMT) in human colorectal cancer metastasis. Gut 62: 1315-1326, 2013.

11. Wang H, Yan C, Shi X, Zheng J, Deng L, Yang L, Yu F, Yang Y and Shao Y: MicroRNA-575 targets BLID to promote growth and invasion of non-small cell lung cancer cells. FEBS Lett 589: 805-811, 2015

12. Zhang Y, Huang W, Ran Y, Xiong Y, Zhong Z, Fan X, Wang Z and Ye Q: miR-582-5p inhibits proliferation of hepatocellular carcinoma by targeting CDK1 and AKT3. Tumour Biol 36: 8309-8316, 2015.

13 Jiang X, Hu C, Arnovitz S,, Bugno J, Yu M, Zuo Z, Chen P, Huang H, Ulrich B, Gurbuxani S, et al: miR-22 has a potent anti-tumour role with therapeutic potential in acute myeloid leukaemia. Nat Commun 7: 11452, 2016.
14. Li Y, Tang X, He Q, Yang X, Ren X, Wen X, Zhang J, Wang Y, Liu N and Ma J: Overexpression of mitochondria mediator gene TRIAP1 by miR-320b loss is associated with progression in nasopharyngeal carcinoma. PLoS Genet 12: e1006183, 2016.

15. Wang H, Cao F, Li X, Miao H, E J, Xing J and Fu CG: miR-320b suppresses cell proliferation by targeting c-Myc in human colorectal cancer cells. BMC Cancer 15: 748, 2015.

16. Batistela MS, Josviak ND, Sulzbach CD and de Souza RL: An overview of circulating cell-free microRNAs as putative biomarkers in Alzheimer's and Parkinson's Diseases. Int J Neurosci: Jul 20, 2016 (Epub ahead of print).

17. Hsu YC, Chang PJ, Ho C, Huang YT, Shih YH, Wang CJ and Lin CL: Protective effects of miR-29a on diabetic glomerular dysfunction by modulation of DKK1/Wnt/ $\beta$-catenin signaling. Sci Rep 6: 30575, 2016.

18. Tana C, Giamberardino MA and Cipollone F: microRNA profiling in atherosclerosis, diabetes and migraine. Ann Med 49: 93-105, 2017.

19. Bao MH, Li JM, Luo HQ, Tang L, Lv QL, Li GY and Zhou HH: NF- $\kappa$ B-regulated miR-99a modulates endothelial cell inflammation. Mediators Inflamm 2016: 5308170, 2016.

20. Ke Y,Zhao W, Xiong J and CaoR: miR-149 inhibits non-small-cell lung cancer cells EMT by targeting FOXM1. Biochem Res Int 2013: 506731, 2013.

21. Cui F, Wang S, Lao I, Zhou C, Kong H, Bayaxi N, Li J, Chen Q, Zhu T and Zhu H: miR-375 inhibits the invasion and metastasis of colorectal cancer via targeting SP1 and regulating EMT-associated genes. Oncol Rep 36: 487-493, 2016.

22. Shi H, Ji Y, Zhang D, Liu Y and Fang P: MiR-135a inhibits migration and invasion and regulates EMT-related marker genes by targeting KLF8 in lung cancer cells. Biochem Biophys Res Commun 465: 125-130, 2015.

23. Babae N, Bourajjaj M, Liu Y, Van Beijnum JR, Cerisoli F, Scaria PV, Verheul M, Van Berkel MP, Pieters EH, Van Haastert RJ, et al: Systemic miRNA-7 delivery inhibits tumor angiogenesis and growth in murine xenograft glioblastoma. Oncotarget 5: 6687-6700, 2014.

24. Yuan J, Xiao G, Peng G, Liu D, Wang Z, Liao Y, Liu Q, Wu M and Yuan X: miRNA-125a-5p inhibits glioblastoma cell proliferation and promotes cell differentiation by targeting TAZ. Biochem Biophys Res Commun 457: 171-176, 2015.

25. Varamo C, Occelli M, Vivenza D, Merlano M and Lo Nigro C: MicroRNAs role as potential biomarkers and key regulators in melanoma. Genes Chromosomes Cancer 56: 3-10, 2017.

26. Ebrahimi S, Ghasemi F, Hassanian SM, Shahidsales S, Maftouh M, Akbarzade H, Parizadeh SA, Hassanian SM and Avan A: Circulating microRNAs as novel potential diagnostic and prognosis biomarkers in pancreatic cancer. Curr Pharm Des 22: 6444-6450, 2016.

27. Kurozumi A, Goto Y, Okato A, Ichikawa T and Seki N: Aberrantly expressed microRNAs in bladder cancer and renal cell carcinoma. J Hum Genet 62: 49-56, 2017.

28. Yan S, Wang T, Huang S, Di Y, Huang Y, Liu X, Luo Z, Han W and An B: Differential expression of microRNAs in plasma of patients with prediabetes and newly diagnosed type 2 diabetes. Acta Diabetol 53: 693-702, 2016.

29. Zhang R, Qin Y, Zhu G, Li Y and Xue J: Low serum miR-320b expression as a novel indicator of carotid atherosclerosis. J Clin Neurosci 33: 252-258, 2016

30. Qin W, Tang Y, Yang N, Wei X and Wu J: Potential role of circulating microRNAs as a biomarker for unexplained recurrent spontaneous abortion. Fertil Steril 105: 1247-1254.e3, 2016.

31. Tadano T, Kakuta Y, Hamada S, Shimodaira Y, Kuroha M, Kawakami Y, Kimura T, Shiga H, Endo K, Masamune A, et al: MicroRNA-320 family is downregulated in colorectal adenoma and affects tumor proliferation by targeting CDK6. World J Gastrointest Oncol 8: 532-542, 2016.

32. Zhou J, Zhang M, Huang Y, Feng L, Chen H, Hu Y, Chen H, Zhang K, Zheng L and Zheng S: MicroRNA-320b promotes colorectal cancer proliferation and invasion by competing with its homologous microRNA-320a. Cancer Lett 356B: 669-675, 2015. 